\title{
CONSTRUCTION OF HILBERT TRANSFORM PAIRS OF WAVELET BASES AND OPTIMAL TIME-FREQUENCY LOCALIZATION
}

\author{
Kunal N. Chaudhury and Michael Unser \\ Biomedical Imaging Group, Ecole Polytechnique Fédérale de Lausanne (EPFL), Switzerland
}

\begin{abstract}
We propose a novel method of constructing exact Hilbert transform (HT) pairs of wavelet bases using fractional Bsplines and state necessary and sufficient conditions for generating such wavelet pairs. In particular, we demonstrate how HT pairs of biorthogonal wavelet bases of $L^{2}(\mathbb{R})$ can be constructed using well-localized scaling functions with identical Riesz bounds. Finally, we illustrate this concept by constructing a family of analytic Gabor-like wavelets that exhibit near optimal time-frequency localization.
\end{abstract}

Index Terms - B-spline, Hilbert transform, Biorthogonal wavelet basis, Time-frequency localization, Gabor function.

\section{INTRODUCTION}

Kingsbury [1] introduced the dual-tree complex wavelet transform and demonstrated its use in a number of applications. There is now good evidence that these complex wavelets have better shift invariance property than the traditional wavelet bases and tend to perform better in a variety of tasks like denoising, texture analysis and deconvolution.

Selesnick [2] made the crucial observation that the dualtree wavelets form an approximate HT pair. He derived a phase relation between the scaling filters that ensures exact HT pair correspondence while he also noted that this correspondence cannot be achieved exactly using rational filterbanks.

In this paper, we propose a method for constructing $e x$ act HT pair of wavelets, in general, and B-spline wavelets in particular. The theoretical difficulty that exact HT pair of wavelets cannot be of finite support is overcome using fractional B-splines [3] that are closed under fractional differentiation including the HT. Moreover, B-spline wavelets have attractive localization properties [4] that can be used advantageously to design Gabor-like wavelets.

The paper is organized as follows. In Section 2 we recall some fundamental definitions and properties of the HT and the fractional B-splines. In Section 3, we derive the necessary and sufficient conditions for constructing wavelet bases that form a HT pair and extend this result to the design of HT pairs

This work was supported in part by the Swiss National Science Foundation under grant 200020-109415 of general biorthogonal wavelet bases of $L^{2}(\mathbb{R})$. In Section 4, we demonstrate the construction of Gabor-like wavelets using HT pairs of semi-orthogonal B-spline wavelets.

\section{HILBERT TRANSFORM AND B-SPLINES}

Definition 2.1 The Hilbert transform $\mathcal{H}: L^{2}(\mathbb{R}) \rightarrow L^{2}(\mathbb{R})$ is defined as

$$
\mathcal{H}\{f\}=\mathcal{F}^{-1}\{-j \operatorname{sign}(\omega) \hat{f}(\omega)\}
$$

where $\hat{f}=\mathcal{F}\{f\}$ is the $L^{2}(\mathbb{R})$-Fourier transform of $f$.

In the definition, $-j \operatorname{sign}(\omega)$ represents the frequency response of the HT operator. The corresponding impulse response is given by $\mathcal{H}\{\delta\}(x)=\mathrm{pv}(1 / \pi x)$ (in the sense of distributions) [5], which indicates the non-local nature of the operator; thus the HT of a compactly supported wavelet is necessarily of infinite support.

A direct implication of (1) is that the HT operator is unitary. Therefore, if $\left\{\psi_{i, k}\right\}_{(i, k) \in \mathbb{Z}^{2}}$ and $\left\{\tilde{\psi}_{j, l}\right\}_{(j, l) \in \mathbb{Z}^{2}}$ form the primal and dual wavelets of a biorthogonal wavelet basis of $L^{2}(\mathbb{R})$, such that $\left\langle\psi_{i, k}, \tilde{\psi}_{j, l}\right\rangle=\delta_{i-j, k-l}$, then by the unitary property of the HT, we have

$$
\left\langle\mathcal{H}\left\{\psi_{i, k}\right\}, \mathcal{H}\left\{\tilde{\psi}_{j, l}\right\}\right\rangle=\left\langle\psi_{i, k}, \tilde{\psi}_{j, l}\right\rangle=\delta_{i-j, k-l}
$$

implying that $\left\{\mathcal{H}\left\{\psi_{i, k}\right\}\right\}_{(i, k) \in \mathbb{Z}^{2}}$ and $\left\{\mathcal{H}\left\{\tilde{\psi}_{j, l}\right\}\right\}_{(j, l) \in \mathbb{Z}^{2}}$ form a biorthogonal wavelet basis of $L^{2}(\mathbb{R})$ as well.

Definition 2.2 The fractional B-spline $\beta_{\tau}^{\alpha}$ of degree $\alpha \in \mathbb{R}_{0}^{+}$ and shift $\tau \in \mathbb{R}$ is specified by its Fourier transform

$$
\hat{\beta}_{\tau}^{\alpha}(\omega)=\left(\frac{e^{j \omega}-1}{j \omega}\right)^{\frac{\alpha+1}{2}-\tau}\left(\frac{1-e^{-j \omega}}{j \omega}\right)^{\frac{\alpha+1}{2}+\tau}
$$

The parameters $\alpha$ and $\tau$ control the width and the average group delay, respectively. Importantly, the fractional Bsplines satisfy the three admissibility conditions for a scaling function to generate a valid multiresolution of $L^{2}(\mathbb{R})$ [3]; they are in general not compactly supported (except for integer degrees), but they decay like $\mathcal{O}\left(1 /|x|^{\alpha+2}\right)$ which ensures their inclusion in $L^{1}(\mathbb{R}) \cap L^{2}(\mathbb{R})$ [3].

We finally introduce the family of fractional finitedifference operators as a generalization of the finite-difference operator. 
Definition 2.3 The fractional finite-difference operator $\Delta_{\tau}^{\alpha}$ : $L^{2}(\mathbb{R}) \rightarrow L^{2}(\mathbb{R})$ is defined as

$$
\Delta_{\tau}^{\alpha}\{f\}=\mathcal{F}^{-1}\{\overbrace{\left(1-e^{-j \omega}\right)^{\frac{\alpha}{2}+\tau}\left(1-e^{j \omega}\right)^{\frac{\alpha}{2}-\tau}}^{\mathcal{D}_{\tau}^{\alpha}\left(e^{j \omega}\right)} \hat{f}(\omega)\}
$$

where $\alpha \in \mathbb{R}_{0}^{+}$gives the order of the finite-difference and $\tau \in \mathbb{R}$ is an adjustable phase factor.

Since the frequency response, $\mathcal{D}_{\tau}^{\alpha}\left(e^{j \omega}\right)$, is $2 \pi$-periodic, this operator also corresponds to a digital filter $\left\{d_{\tau}^{\alpha}[k]\right\}_{k \in \mathbb{Z}}$ with the correspondence $\Delta_{\tau}^{\alpha}\{f\}=\sum_{k \in \mathbb{Z}} d_{\tau}^{\alpha}[k] f(\cdot-k)$.

The finite-difference operator that is especially relevant in this context corresponds to $\alpha=0$ and $\tau=-1 / 2$. The corresponding frequency response reduces ${ }^{\dagger}$ to $\mathcal{D}_{-\frac{1}{2}}^{0}\left(e^{j \omega}\right)=$ $-j \operatorname{sign}(\omega) e^{j \omega / 2}$, for $\omega \in(-\pi, \pi]$, which is similar to the all-pass frequency response of the HT operator. The corresponding digital filter $d_{-\frac{1}{2}}^{0}$ also resembles the usual discrete HT filters:

Proposition 2.4 The digital filter corresponding to the finitedifference operator $\Delta_{-\frac{1}{2}}^{0}$ is specified by

$$
d_{-\frac{1}{2}}^{0}[k]=\frac{1}{\pi\left(k+\frac{1}{2}\right)}, k \in \mathbb{Z}
$$

Based on this finite-difference filter, we have a closed form expression for the HT of the fractional B-spline $\beta_{\tau}^{\alpha}$ which happens to be in the linear span of fractional B-splines of the same degree $\alpha$ but modified shift $\left(\tau+\frac{1}{2}\right)$.

Proposition 2.5 The Hilbert transform of a fractional Bspline can be expressed as

$$
\mathcal{H}\left\{\beta_{\tau}^{\alpha}\right\}=\Delta_{-\frac{1}{2}}^{0}\left\{\beta_{\tau+\frac{1}{2}}^{\alpha}\right\}=\sum_{k \in \mathbb{Z}} d_{-\frac{1}{2}}^{0}[k] \beta_{\tau+\frac{1}{2}}^{\alpha}(\cdot-k)
$$

Note that digital filter $d_{-\frac{1}{2}}^{0}$ is a "half-shifted" version of the usual discrete Hilbert filter and acts as an unitary operator $\Delta_{-\frac{1}{2}}^{0}: L^{2}(\mathbb{R}) \rightarrow L^{2}(\mathbb{R})$, when applied to functions or as an unitary convolution operator $d_{-\frac{1}{2}}^{0}: l_{2}(\mathbb{Z}) \rightarrow l_{2}(\mathbb{Z})$, when applied to sequences.

Remark: The theoretical difficulty with the HT stems from the fact that its frequency response has a singularity at $\omega=$ 0 , which results in a poor decay of the transformed output. The remarkable feature of (6) is that we have been able to express the slowly decaying HT as a linear combination of the better-behaved B-splines. Specifically, $d_{-\frac{1}{2}}^{0}$ only decays like $\mathcal{O}(1 /|k|)$ whereas $\beta_{\tau+\frac{1}{2}}^{\alpha}$ decays as $\mathcal{O}\left(1 /|x|^{\alpha+2}\right)$.

Thus by expressing the HT using shifted B-splines as in (6), we have in effect moved this singularity onto the digital

\footnotetext{
${ }^{\dagger}$ The (principal value) fractional power of a complex number $z$ is defined as $z^{\gamma}=|z|^{\gamma} e^{j \gamma \arg (z)}$ corresponding to the principal $\operatorname{argument} \arg (z) \in$ $(-\pi, \pi]$. Specifically, $\left(\prod_{k} z_{k}\right)^{\gamma}=\prod_{k} z_{k}^{\gamma}$ only if $\sum_{k} \arg \left(z_{k}\right) \in(-\pi, \pi]$.
}

filter. In the sequel, we will see that this filter will in turn be applied to the wavelets. There, its effect is much more innocuous because $\hat{\psi}(\omega)=0$ around the origin and hence the damage to the decay of the wavelets is minimal.

The shift parameter $\tau$ only affects the phase of the Fourier transform of the fractional B-spline and the corresponding scaling filter [3]. The relation between the scaling filters of fractional B-splines which have a difference of $1 / 2$ in their shift parameter is especially enlightening:

\section{Proposition 2.6}

$$
H_{\tau+\frac{1}{2}}^{\alpha}\left(e^{j \omega}\right)=e^{-j \omega / 2} H_{\tau}^{\alpha}\left(e^{j \omega}\right), \text { for } \omega \in(-\pi, \pi]
$$

In effect, the former filter is a "half-sample" shift of the latter. In other words, if we construct a unique bandlimited function $h_{\tau}^{\alpha}(x)=\sum_{k \in \mathbb{Z}} h_{\tau}^{\alpha}[k] \operatorname{sinc}(x-k)$, such that $\left.h_{\tau}^{\alpha}(x)\right|_{x=k}=$ $h_{\tau}^{\alpha}[k]$, then the above relation implies that $h_{\tau+\frac{1}{2}}^{\alpha}[k]=h_{\tau}^{\alpha}(k-$ $\left.\frac{1}{2}\right), k \in \mathbb{Z}$, i.e. each filter provides the bandlimited interpolation of the other mid-way between its samples.

\section{HT PAIR OF WAVELET BASES}

A fundamental result in wavelet theory is that it is always possible to express a valid scaling function as a convolution between a fractional B-spline and a distribution [6]. The original theorem involves causal B-splines, but the result can readily be extended to the more general $(\alpha, \tau)$ B-splines.

Theorem 3.1 (B-spline Factorization) $\varphi$ is a valid scaling function of order $\alpha+1$ if and only if its Fourier transform $\hat{\varphi}$ can be factorized as $\hat{\varphi}(\omega)=\hat{\beta}_{\tau}^{\alpha}(\omega) \cdot \hat{\varphi}_{0}(\omega)$, for some $\tau \in \mathbb{R}$, where $\hat{\varphi}_{0}(\omega)$ is a true function of $\omega$ bounded on every closed interval with $\hat{\varphi}_{0}(0)=1$.

In the signal domain, this corresponds to a well-defined convolution between a fractional B-spline and a tempered distribution, i.e., $\varphi=\beta_{\tau}^{\alpha} * \varphi_{0}$ where $\varphi_{0} \in \mathcal{S}^{\prime}(\mathbb{R})[6]$.

In the present context, we are particularly interested in the scaling functions

$$
\varphi=\beta_{\tau}^{\alpha} * \varphi_{0}, \varphi^{\prime}=\beta_{\tau+\frac{1}{2}}^{\alpha} * \varphi_{0}
$$

generated by the underlying fractional B-splines $\beta_{\tau}^{\alpha}$ and $\beta_{\tau+\frac{1}{2}}^{\alpha}$ of the same order but with a relative half-shift. Importantly, $\varphi$ and $\varphi^{\prime}$ are both valid scaling functions of order $\alpha+1$, i.e., they generate Riesz bases, satisfy the two-scale relation, as well as the partition of unity condition.

The HT of a wavelet is also a wavelet in a well-defined sense; i.e, if $\psi$ is a wavelet whose dilation-shifts $\left\{2^{j / 2} \psi\left(2^{j}\right.\right.$. $-k)\}_{(j, k) \in \mathbb{Z}^{2}}$ forms a Riesz basis of $L^{2}(\mathbb{R})$ then the same is true for $\left\{2^{j / 2} \mathcal{H}\{\psi\}\left(2^{j} \cdot-k\right)\right\}_{(j, k) \in \mathbb{Z}^{2}}$. This directly follows from the unitary nature of the HT operator. Based on Theorem (3.1), we state the following necessary and sufficient condition for generating HT pair of wavelets: 
Theorem 3.2 (HT Pair of Wavelets) Let $\varphi \in L^{2}(\mathbb{R})$ be a valid scaling function of order $\alpha+1$ and $\psi$ be a corresponding wavelet specified by

$$
\psi=\sum_{k \in \mathbb{Z}} g[k] \varphi(2 \cdot-k)
$$

Let $\varphi=\beta_{\tau}^{\alpha} * \varphi_{0}$, with $\varphi_{0} \in \mathcal{S}^{\prime}(\mathbb{R})$, be a factorization of $\varphi$ for some $\tau \in \mathbb{R}$. Consider the valid scaling function $\varphi^{\prime}:=$ $\beta_{\tau+\frac{1}{2}}^{\alpha} * \varphi_{0}$ of order $\alpha+1$ and a corresponding wavelet $\psi^{\prime}$ specified by

$$
\psi^{\prime}=\sum_{k \in \mathbb{Z}} g^{\prime}[k] \varphi^{\prime}(2 \cdot-k)
$$

Then $\psi^{\prime}=\mathcal{H}\{\psi\}$ if and only if $g^{\prime}=d_{-\frac{1}{2}}^{0} * g$.

Moreover,

(a) Both $\varphi$ and $\varphi^{\prime}$ have the same Riesz bounds, and

(b) The frequency response $H\left(e^{j \omega}\right)$ and $H^{\prime}\left(e^{j \omega}\right)$ of the scaling filters corresponding to $\varphi$ and $\varphi^{\prime}$ (resp.) are related as

$$
H^{\prime}\left(e^{j \omega}\right)=e^{-j \omega / 2} H\left(e^{j \omega}\right), \omega \in(-\pi, \pi]
$$

Remark: It is critical to note that although the HT $\psi^{\prime}=$ $\mathcal{H}\{\psi\}$ of a given wavelet $\psi$ is unique, the scaling function $\varphi^{\prime}$ and the corresponding $g^{\prime}$ generating $\psi^{\prime}$ is by no means unique. For instance, the trivial choice of $\varphi^{\prime}=\mathcal{H}\{\varphi\}$ and $g^{\prime}=g$ is sufficient to ensure $\psi^{\prime}=\mathcal{H}\{\psi\}$. The necessary and sufficient condition in Theorem (3.2) holds only for our preferred choice of the scaling function $\varphi^{\prime}=\beta_{\tau+\frac{1}{2}}^{\alpha} * \varphi_{0}$. Moreover, this particular choice of scaling function over the more direct one $\varphi^{\prime}=\mathcal{H}\{\varphi\}$ is justified because:

(1) $\mathcal{H}\{\varphi\}$ has poor decay properties and is not in $L^{1}(\mathbb{R})$,

(2) $\mathcal{H}\{\varphi\}$ does not satisfy the partition-of-unity condition as $\widehat{\mathcal{H}\{\varphi\}}(0) \neq 1$, and is therefore not admissible in the conventional sense.

A biorthogonal wavelet basis of $L^{2}(\mathbb{R})$ involves two multiresolution analyses $\left\{2^{j / 2} \tilde{\varphi}\left(2^{j} x-k\right):(j, k) \in \mathbb{Z}^{2}\right\}$ and $\left\{2^{j / 2} \varphi\left(2^{j} x-k\right):(j, k) \in \mathbb{Z}^{2}\right\}$, where $\tilde{\varphi}$ and $\varphi$ are valid scaling functions of order $(\tilde{N}+1, N+1)$. The corresponding wavelets $(\tilde{\psi}, \psi)$ satisfy the biorthogonality condition $\langle\tilde{\psi}, \psi(\cdot-k)\rangle=\delta_{k}, k \in \mathbb{Z}[6]$.

As discussed earlier, we can represent such scaling functions in terms of the underlying B-splines as $\tilde{\varphi}=\beta_{\tilde{\tau}}^{\tilde{N}} * \tilde{\varphi}_{0}$ and $\varphi=\beta_{\tau}^{N} * \varphi_{0}$, where $\tilde{\varphi}_{0}, \varphi_{0} \in \mathcal{S}^{\prime}(\mathbb{R})$ with $\hat{\tilde{\varphi}}_{0}(0)=\hat{\varphi}_{0}(0)=$ 1. Based on Theorem 3.2, we propose the following construction of HT pairs of biorthogonal wavelet bases of $L^{2}(\mathbb{R})$ :

Corollary 3.3 (HT Pairs of Biorthogonal Wavelet Bases) Let $\tilde{\varphi}$ and $\varphi$ define a biorthogonal wavelet basis of $L^{2}(\mathbb{R})$ of $\operatorname{order}(\tilde{N}+1, N+1)$ with associated wavelets

$$
\tilde{\psi}=\sum_{k \in \mathbb{Z}} \tilde{g}[k] \tilde{\varphi}(2 \cdot-k), \psi=\sum_{k \in \mathbb{Z}} g[k] \varphi(2 \cdot-k)
$$

Let $\tilde{\varphi}=\beta_{\tilde{\tau}}^{\tilde{N}} * \tilde{\varphi}_{0}$ be a factorization of $\tilde{\varphi}$ for some $\tilde{\tau} \in \mathbb{R}$. Similarly, let $\varphi=\beta_{\tau}^{N} * \varphi_{0}$ be a factorization of $\varphi$ for some $\tau \in \mathbb{R}$.
Now, consider the multiresolution analyses generated by the valid scaling functions $\tilde{\varphi}^{\prime}:=\beta_{\tilde{\tau}+\frac{1}{2}}^{\tilde{N}} * \tilde{\varphi}_{0}$ and $\varphi^{\prime}:=\beta_{\tau+\frac{1}{2}}^{N} *$ $\varphi_{0}$ with corresponding wavelets

$$
\tilde{\psi}^{\prime}=\sum_{k \in \mathbb{Z}} \tilde{g}^{\prime}[k] \tilde{\varphi}^{\prime}(2 \cdot-k), \psi^{\prime}=\sum_{k \in \mathbb{Z}} g^{\prime}[k] \varphi^{\prime}(2 \cdot-k)
$$

Then

(a) $\tilde{\psi}^{\prime}=\mathcal{H}\{\tilde{\psi}\}$ and $\psi^{\prime}=\mathcal{H}\{\psi\}$, and

(b) $\left(\tilde{\psi}^{\prime}, \psi^{\prime}\right)$ constitutes a valid biorthogonal wavelet basis of $L^{2}(\mathbb{R})$ satisfying $\left\langle\tilde{\psi}^{\prime}, \psi^{\prime}(\cdot-k)\right\rangle=\delta_{k}, k \in \mathbb{Z}$,

if and only if $\tilde{g}^{\prime}=d_{-\frac{1}{2}}^{0} * \tilde{g}$ and $g^{\prime}=d_{-\frac{1}{2}}^{0} * g$.

The proposed construction has the following additional properties:

- The two biorthogonal systems are of the same order $(\tilde{N}+1, N+1)$ and have the same Riesz bounds.

- If the scaling functions $(\tilde{\varphi}, \varphi)$ satisfy the biorthogonality relation $\langle\tilde{\varphi}, \varphi(\cdot-k)\rangle=\delta_{k}, k \in \mathbb{Z}$, then so do $\left(\tilde{\varphi}^{\prime}, \varphi^{\prime}\right)$.

- The scaling filters on both the analysis and synthesis side are "half-sample shifted" versions of one another

$$
\begin{aligned}
H^{\prime}\left(e^{j \omega}\right) & =e^{-j \omega / 2} H\left(e^{j \omega}\right), \\
\tilde{H}^{\prime}\left(e^{j \omega}\right) & =e^{-j \omega / 2} \tilde{H}\left(e^{j \omega}\right), \omega \in(-\pi, \pi]
\end{aligned}
$$

- Finally, if the analysis and synthesis filters of the original biorthogonal system satisfy the perfect reconstruction conditions then so do the filters of the HT pairs.

\section{GABOR-LIKE WAVELETS}

As a practical application of the results developed so far, we demonstrate the construction of HT pair of semi-orthogonal B-spline wavelets that are better localized in space than their orthonormal counterpart [7].

Starting from the scaling function $\varphi=\beta_{\tau}^{\alpha}$, the transfer function of the "shortest" (most localized) wavelet filter that generates the semi-orthogonal wavelet $\psi_{\tau}^{\alpha}$ with the semiorthogonality condition $\left\langle\beta_{\tau}^{\alpha}(\cdot), \psi_{\tau}^{\alpha}(\cdot-k)\right\rangle=0$ for all $k \in \mathbb{Z}$, is then given by

$$
G(z)=z A(-z) H\left(-z^{-1}\right)
$$

where $H(z)$ and $A(z)=\sum_{k \in \mathbb{Z}}\langle\varphi, \varphi(\cdot-k)\rangle z^{-k}$ are the scaling and autocorrelation filter [7] of the scaling function $\varphi$, respectively. The associated biorthogonal system comprises of the (unique) dual-spline scaling function $\tilde{\varphi}=\AA_{\tau}^{\alpha}$ and the dual wavelet $\tilde{\psi}_{\tau}^{\alpha}$, with corresponding scaling and wavelet filter $\tilde{h}$ and $\tilde{g}$, respectively [7]. Based on Corollary (3.3), we construct the following HT pair of B-spline wavelet bases: 


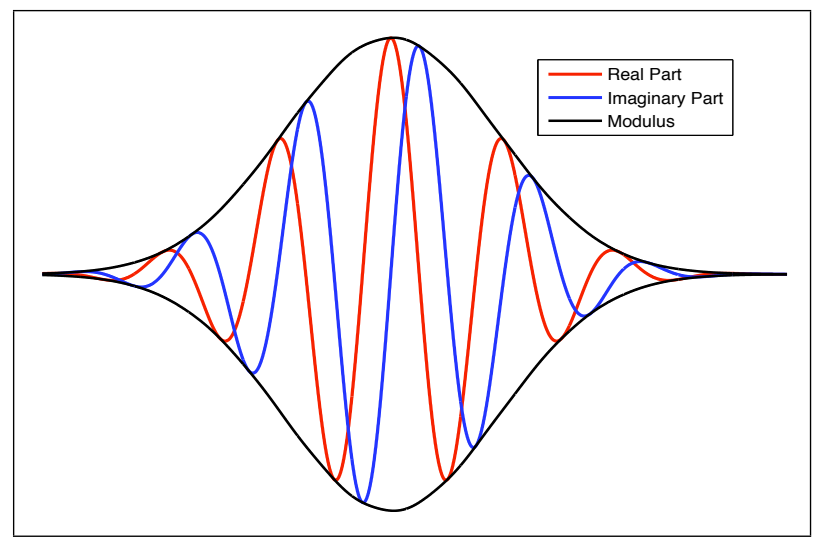

Fig. 1. Gabor-like complex wavelet using HT pairs of Bspline wavelets. Real Part: $\psi_{\tau}^{\alpha}$, Imaginary Part: $\psi_{\tau+\frac{1}{2}}^{\alpha}$.

Proposition 4.1 (HT Pair of B-spline Wavelets) Consider the new biorthogonal system with scaling function $\varphi^{\prime}=\beta_{\tau+\frac{1}{2}}^{\alpha}$ and corresponding B-spline wavelet $\psi_{\tau+\frac{1}{2}}^{\alpha}$. Also, let $\tilde{\varphi}^{\prime}=$ $\stackrel{\circ}{\beta}_{\tau+\frac{1}{2}}^{\alpha}$ and $\tilde{\psi}_{\tau+\frac{1}{2}}^{\alpha}$ be the corresponding dual-spline scaling function and wavelet, respectively.

Then $\psi_{\tau+\frac{1}{2}}^{\alpha}=\mathcal{H}\left\{\psi_{\tau}^{\alpha}\right\}$ and $\tilde{\psi}_{\tau+\frac{1}{2}}^{\alpha}=\mathcal{H}\left\{\tilde{\psi}_{\tau}^{\alpha}\right\}$.

Proof. The B-spline scaling functions are trivially factorized into $\varphi=\beta_{\tau}^{\alpha} * \varphi_{0}$ and $\varphi^{\prime}=\beta_{\tau+\frac{1}{2}}^{\alpha} * \varphi_{0}$, where $\varphi_{0}=\delta$. Similarly, the dual-spline scaling functions are factorized into $\tilde{\varphi}=$ $\beta_{\tau}^{\alpha} * \tilde{\varphi}_{0}$ and $\tilde{\varphi}^{\prime}=\beta_{\tau+\frac{1}{2}}^{\alpha} * \tilde{\varphi}_{0}$, with $\tilde{\varphi}_{0}:=\sum_{k \in \mathbb{Z}} q^{\alpha}[k] \delta(\cdot-k)$ where $\sum_{k \in \mathbb{Z}} q^{\alpha}[k] e^{-j \omega k}=1 / A^{\alpha}\left(e^{j \omega}\right)$. Here, we have used the fact that $A^{\alpha}\left(e^{j \omega}\right)$ is independent of the shift-parameter $\tau$.

Finally, we verify that the wavelet filters, which are related to the corresponding scaling filters via (15), satisfy the sufficiency conditions $\tilde{g}^{\prime}=d_{-\frac{1}{2}}^{0} * \tilde{g}$ and $g^{\prime}=d_{-\frac{1}{2}}^{0} * g$. Hence, as per Corollary 3.3, the proposition follows.

As the degree $\alpha$ increases, the semi-orthogonal B-spline wavelets converge pointwise to the real part of a Gabor function (modulated Gaussian) [4]. This is of particular importance as the Gabor functions are known to have optimal timefrequency localization property in the sense of Heisenberg's uncertainty principle. By appropriately adapting the corresponding proof in [4], we can shown that the fractional Bspline wavelet $\psi_{\tau}^{\alpha}$ asymptotically convergences to the real part of of the Gabor function

$\psi_{\tau}^{\alpha}(x) \underset{\alpha \rightarrow+\infty}{\approx} \frac{2 M_{0}^{\alpha+1} \Delta \omega_{0}}{\sqrt{2 \pi(\alpha+1)}} e^{-\frac{\Delta \omega_{0}^{2}\left(x-\frac{1}{2}\right)^{2}}{2(\alpha+1)}} \cos \left(\omega_{0} x-\frac{\omega_{0}}{2}-\pi \tau\right)$ where, $M_{0}=0.670, \omega_{0}=-5.142$ and $\Delta \omega_{0}=2.670$. Finally, using the fact $\psi_{\tau+\frac{1}{2}}^{\alpha}=\mathcal{H}\left\{\psi_{\tau}^{\alpha}\right\}$, we have the following convergence result.

Proposition 4.2 (Gabor-like Wavelet) The analytic B-spline wavelet $\Psi_{\tau}^{\alpha}:=\psi_{\tau}^{\alpha}+j \psi_{\tau+\frac{1}{2}}^{\alpha}$ asymptotically converges to a
Gabor-like function, i.e.,

$$
\Psi_{\tau}^{\alpha}(x) \underset{\alpha \rightarrow+\infty}{\approx} \frac{2 M_{0}^{\alpha+1} \Delta \omega_{0}}{\sqrt{2 \pi(\alpha+1)}} e^{-\frac{\Delta \omega_{0}^{2}(x-1 / 2)^{2}}{2(\alpha+1)}} e^{j\left(\omega_{0} x-\frac{\omega_{0}}{2}-\pi \tau\right)}
$$

As far as the rate of convergence is concerned, the joint time-frequency resolution of the complex cubic B-spline wavelet $(\alpha=3)$ is already within $3 \%$ of the limit specified by the uncertainity principle. Fig. 1 shows such a Gaborlike wavelet generated using HT pair of B-spline wavelets of degree $\alpha=6$. Also, shown in the figure is the magnitude envelope $\left|\Psi_{\tau}^{\alpha}\right|$ of the complex wavelet which closely matches the well-localized Gaussian window of the Gabor function. To the best of our knowledge, these are the best localized wavelets that have been constructed so far.

\section{CONCLUSION}

We have demonstrated the construction of exact HT pairs of biorthogonal wavelet bases, in general, and B-spline wavelets in particular. We have introduced a new family of analytic Bspline wavelets and shown that they asymptotically converge to Gabor functions with optimal time-frequency localization. As will be demonstrated elsewhere, this construction can be extended to the design of 2D wavelets with both directional and optimal localization properties.

\section{REFERENCES}

[1] N.G. Kingsbury, "Complex wavelets for shift invariant analysis and filtering of signals," Journal of Applied and Computational Harmonic Analysis, vol. 10, no. 3, pp. 234-253, May 2001.

[2] I.W. Selesnick, "Hilbert transform pairs of wavelet bases," IEEE Signal Processing Letters, vol. 8, no. 6, pp. 170-173, 2001.

[3] T. Blu and M. Unser, "A complete family of scaling functions: The $(\alpha, \tau)$-fractional splines," Proceedings: ICASSP, vol. VI, pp. 421-424, April 6-10 2003.

[4] M. Unser, A. Aldroubi, and M. Eden, "On the asymptotic convergence of B-spline wavelets to Gabor functions," IEEE Trans. Information Theory, vol. 38, no. 2, pp. 864-872, March 1992.

[5] J. J. Benedetto, Harmonic Analysis and Applications, CRC Press, 1996.

[6] M. Unser and T. Blu, "Wavelet theory demystified," IEEE Transactions on Signal Processing, vol. 51, no. 2, pp. 470-483, February 2003.

[7] M. Unser and T. Blu, "Construction of fractional spline wavelet bases," Proc. SPIE Conf. on Mathematical Imaging: Wavelet Applications in Signal and Image Processing VII, vol. 3813, pp. 422-431, July 1999. 